\title{
Causa de mortalidade materna na região metropolitana I no triênio 2013-2015, Belém, PA
}

\begin{abstract}
RESUMO | Objetivo: estudo sobre a ocorrência de óbitos maternos na região metropolitana de Belém no período de janeiro de 2013 a dezembro de 2015. Método: Trata-se de uma abordagem epidemiológica, descritiva, quantitativa, retrospectiva e documental. Incluindo: óbitos classificados como morte materna por causas obstétricas diretas e indiretas. Sendo excluído: óbitos classificados como acidentais. Por intermédio da estatística descritiva. Resultado: No período de janeiro de 2013 a dezembro de 2015, foram declarados 53 óbitos em 2013, 45 óbitos maternos no ano de 2014 e 40 óbitos em 2015. Os dados identificaram uma razão de mortalidade materna de 160,3 no ano de 2013, 136,0 no ano de 2014 e 121,9 em 2015. Conclusão: A morte materna na região metropolitana I é caracterizada por RMM acima do recomendado pela OMS, evidenciando a necessidade de atenção integral e de qualidade à saúde da gestante, desde o pré-natal até o puerpério, no Estado do Pará.
\end{abstract}

Palavras-chaves: Mortalidade Materna; Saúde da Mulher; Epidemiologia.

\begin{abstract}
Objective: a study on the occurrence of maternal deaths in the metropolitan region of Belém from January 2013 to December 2015. Method: This is an epidemiological, descriptive, quantitative, retrospective and documentary approach. Including: deaths classified as maternal deaths from direct and indirect obstetric causes. Being excluded: deaths classified as accidental. By means of descriptive statistics. Outcome: From January 2013 to December 2015, 53 deaths were reported in 2013, 45 maternal deaths in 2014 and 40 deaths in 2015. The data identified a maternal mortality ratio of 160.3 in the year 2013, 136.0 in 2014 and 121.9 in 2015. Conclusion: Maternal death in the metropolitan region I is characterized by MMR above that recommended by the WHO, evidencing the need for integral attention and quality to the health of the pregnant woman, to the puerperium in the state of Pará.
\end{abstract}

Keywords: Maternal Mortality; Women's Health; Epidemiology.

RESUMEN I Objetivo: Estudio sobre la ocurrencia de muertes maternas en la región metropolitana de Belém en el período de enero de 2013 a diciembre de 2015. Método: Se trata de un abordaje epidemiológico, descriptivo, cuantitativo, retrospectivo y documental. Incluyendo: óbitos clasificados como muerte materna por causas obstétricas directas e indirectas. Si se excluye: muertes clasificadas como accidentales. Por medio de la estadística descriptiva. Resultado: En el período de enero de 2013 a diciembre de 2015, se declararon 53 muertes en 2013, 45 muertes maternas en el año 2014 y 40 muertes en 2015. Los datos identificaron una razón de mortalidad materna de 160,3 en el año 2013, En el año 2014 y 121,9 en 2015. Conclusión: La muerte materna en la región metropolitana I se caracteriza por RMM por encima de lo recomendado por la OMS, evidenciando la necesidad de atención integral y de calidad a la salud de la gestante, desde el preescolar, -natal hasta el puerperio, en el Estado de Pará.

Descriptores: Mortalidad Materna; Salud de la Mujer; Epidemiología.

\section{Elyade Nelly Pires Rocha Camacho}

Enfermeira Obstetra. Professora, Graduação em Enfermagem pela Universidade da Amazônia. Belém (PA). Mestre em Enfermagem e Doutoranda em Doenças Tropicais de Medicina Tropical pela Universidade Federal do Pará (UFPA).

\section{Eliete da Cunha Araújo}

Médica Pediatra. Doutorado em Medicina Tropical pela Fundação Oswaldo Cruz (2007) e Mestrado em Medicina Tropical pela Universidade Federal do Pará (1998). Professora Titular em Pediatria e Diretora Geral do Instituto de Ciências da Saúde da Universidade Federal do Pará-UFPA.

\section{Elisângela da Silva Ferreira}

Enfermeira graduada pela Universidade Federal do Pará (UFPA - 2008), Pós-graduada Lato Senso em Enfermagem em Terapia Intensiva (UFPA). Especialista em Enfermagem Oncológica Modalidade Residência do Hospital Ophir Loyola. Especialista em Enfermagem Obstétrica . Mestre em Biologia dos Agentes Infecciosos e Parasitários - BAIP. Doutoranda do Programa de Engenharia em Recursos Naturais da Amazônia/PRODERNA.

\section{Rubenilson Caldas Valois}

Enfermeiro. Doutorado em Doenças Tropicais pelo Núcleo de Medicina Tropical - UFPA, Mestre em Biologia de Agentes Infecciosos e Parasitários pela Universidade Federal do Pará, especializações em enfermagem obstétrica pela UFPA e hemoterapia e hematologia pela UEPA. Atua como Professor Assistente I da Universidade do Estado do Pará, onde ministro a disciplina estágio supervisionado em Enfermagem Obstétrica.

\section{Andressa Tavares Parente \\ Graduação em Enfermagem pela Universidade do Estado do Pará (2004), Mestrado (2007) e Doutorado em Ciências Ambientais (2016) pela parceria Universidade Federal do Pará, Museo Emilio Goeldi e Embrapa. Atualmente é enfermeira da Fundação Santa Casa de Misericórdia do Pará (setor de neonatologia) e professora adjunta da Universidade Federal do Pará, da atividade curricular de pediatria.}

\section{Fabio Feitosa Camacho}

Psicólogo, Graduação pela Universidade da Amazônia. Belém (PA). Especializando em Psicologia Hospitalar pela UNIBF.

Recebido em: 10/06/2019

Aprovado em: 10/08/2019

INTRODUÇÃO

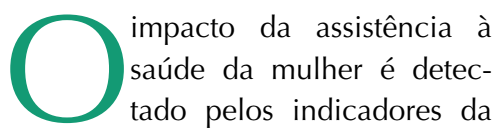
saúde feminina, dentre eles, a Mortalidade Materna, o que está diretamente relacionado com a má qualidade da assistência no pré-natal, no parto e puerpério'. A redução desta mortalidade é uma das principais Metas do Desenvolvimento do Milênio da $\mathrm{ONU}^{2}$.

Um dos objetivos do pacto é identificar os principais fatores de risco que 
contribuem para esse cenário. Sabemos que se relacionam com processo saúde e doença, e por consequência, permitem definir estratégias de prevenção de novas ocorrências. Conhecer o impacto social e a implementação dessa política é importante para o direcionamento dos profissionais de saúde em sua atuação no âmbito da atenção primária ${ }^{3}$.

A morte materna é definida como a morte da gestante ou mulher que esteve em gestação durante as seis semanas (42 dias) anteriores ao óbito, devido a qualquer causa direta ou indiretamente relacionada à gestação e a seu manejo. Mortes maternas são divididas em mortes obstétricas diretas (hemorragia, sepses etc.) e indiretas (doenças já existentes complicadas pela gestação). $\mathrm{O}$ indicador mais utilizado para calcular a mortalidade materna é o número de mortes maternas por 100.000 nascidos vivos, denominado razão de mortalidade materna (RMM). Esta não é uma taxa ou coeficiente, pois o denominador é o número de nascimentos, e o numerador o número de mulheres ${ }^{4}$.

A RMM é o indicador utilizado para medir a mortalidade materna. Contudo, dois problemas para sua correta aferição persistem: a subinformação (através do preenchimento incorreto das causas de morte na declaração de óbito); e o sub-registro (que é a ausência da DO nas bases de dados oficiais). Mesmo em países onde o registro de mortes tem uma cobertura que se aproxima dos $100 \%$, os problemas de subinformação permanecem. Estudos realizados em países desenvolvidos evidenciam que a subestimação no número de mortes devidas à gravidez e suas complicações é uma questão que diz respeito tanto aos países desenvolvidos como aos países em desenvolvimento, embora mais importante nestes últimos ${ }^{5}$.

Entre outras razões, a mortalidade materna se torna um evento que se relaciona ao acesso e à qualidade dos serviços e ações de saúde, sobretudo no campo reprodutivo e sexual, envolvendo aspectos modelares relativos à organização, gestão e cuidados que merecem des- taque quando se objetiva sua superação. Nesse sentido, a realização de estudos sobre a ocorrência de óbitos maternos, agregando mais informações, poderá contribuir para o melhor direcionamento de ações de saúde que possam impactar de forma positiva neste agravo. Frente a este contexto, emergiu a seguinte pergunta da pesquisa: Qual a Razão de Mortalidade Materna (RMM) encontrada na região metropolitana de Belém, ela está abaixo do preconizado pela OMS?

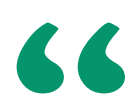

A morte materna é definida como a morte da gestante ou mulher que esteve em gestação durante as seis semanas (42 dias) anteriores ao óbito, devido a qualquer causa direta ou indiretamente relacionada à gestação e a seu
manejo.

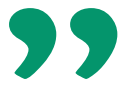

Diante do exposto, o objetivo do presente trabalho consiste em descrever a Razão da Mortalidade Materna na região metropolitana I de Belém, no Estado do Pará, no período de janeiro de 2013 a dezembro de 2015.

\section{MÉTODO}

Trata-se de um estudo quantitativo ecológico, sendo realizado a partir de dados coletados junto a Secretaria de Estado de Saúde Pública do Pará (SESPA), por meio do Sistema de Informação de Mortalidade (SIM).

A amostra foi constituída de um total de 138 óbitos notificados como sendo por causas maternas no período de janeiro de 2013 a dezembro de 2015. Foram incluídas mortes classificadas como materna, que de acordo com o Ministério da Saúde refere-se à óbito de mulheres de 10 a 49 anos, com idade fértil e que evoluíram para óbito no período de janeiro de 2013 a dezembro de 2015. Fizeram parte da pesquisa os óbitos ocorridos durante a gestação, parto ou até 42 dias após o término da mesma e causados por qualquer fator relacionado ou agravado pela gravidez ou por medidas tomadas. Foram excluídas fichas de notificação que se encontrava incompleta, ou preenchida de maneira equivocada, notificado como óbitos acidentais ou relacionado à outras causas que não estejam associadas ao ciclo gravídico.

Este estudo foi enviado a Plataforma Brasil, sendo aprovado no Comitê de Ética e Pesquisa da Universidade Federal do Pará, sob o $N^{\circ}$ do Parecer: 1.308.500 e CAAE: 41727515.0.0000.0018, e por se tratar de dados secundários, de acesso livre ao público, não fez-se necessário o uso de Termo de Consentimento Livre Esclarecido.

Posteriormente foi realizado o cálculo da razão de mortalidade materna bruta quanto a separada por ano. Além disso, foi calculada a razão de mortalidade específica relacionada às causas de morte materna no Pará. Todos os cálculos foram realizados com base no que foi conceituado pelo MS. Para a análise estatística, foi utilizado do Microsoft Excel $2007^{\circledR}$, para confecção de banco de dados, tabelas e gráficos.

$N^{\circ}$ ÓBITOS MATERNOS

$\mathrm{N}^{\circ}$ NASCIDOS VIVOS 
Camacho, E.N.P.R; Araújo, E.C.; Ferreira, E.S.; Valois, R.C.; Parente, A.T.; Camacho, FF.; Causa de mortalidade matema na regiäo metropolitana I no triênio 2013-2015, Belém, PA

Tabela 1 - Óbitos maternos em números absolutos (n), número de nascidos vivos (NV) e a Razão de Mortalidade Materna (RMM) ocorridos na região metropolitana I do estado do Pará, no período de janeiro de 2013 a dezembro de 2015.

\begin{tabular}{lccc} 
Ano & $\mathbf{n}$ & NV & RMM \\
2013 & 53 & 33061 & 160,3 \\
\hline 2014 & 45 & 33100 & 136,0 \\
\hline 2015 & 40 & 32807 & 121,9 \\
\hline $2013-2015$ & 138 & 98968 & 139,4
\end{tabular}

Fonte: Dados da pesquisa.

Figura 01: Razão de mortalidade materna (por 100 mil nascidos vivos), ocorridos na região metropolitana I do estado do Pará, no periodo de janeiro de 2013 a dezembro de 2015.

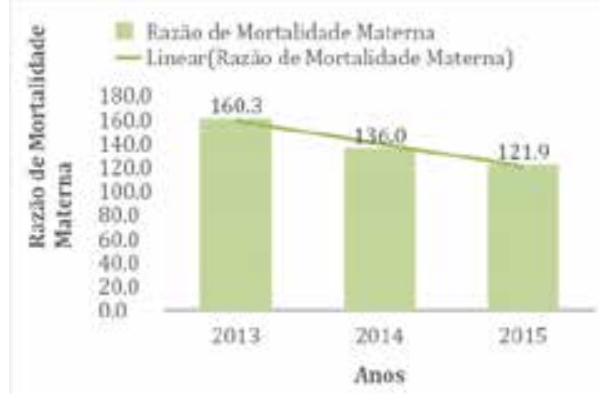

Tabela 2 - Razão de mortalidade materna por causas obstétricas diretas e indiretas (por 100 mil nascidos vivos), ocorridos na região metropolitana I do estado do Pará, no período de janeiro de 2013 a dezembro de 2015.

\begin{tabular}{lccccccccc} 
Ano & \multicolumn{2}{c}{2013} & 2014 & 2015 & \multicolumn{3}{c}{ Variação } \\
& n & RMM & N & RMM & n & RMM & $\begin{array}{c}2014- \\
2013\end{array}$ & $2015-$ & $2015-$ \\
& & & & & & & & & \\
$\begin{array}{l}\text { Morte Materna Obstétrica } \\
\text { Direta }\end{array}$ & 30 & 90,7 & 26 & 78,6 & 24 & 73,2 & & & \\
$\begin{array}{l}\text { Morte Materna Obstétrica } \\
\text { Indireta }\end{array}$ & 23 & 69,6 & 19 & 57,4 & 16 & 48,8 & & & \\
TOTAL & 53 & 160,3 & 45 & 136,0 & 40 & 121,9 & 15,19 & 10,32 & 23,94 \\
\hline
\end{tabular}

Fonte: Dados da pesquisa.

Figura 02: Razão de mortalidade materna por causas obstétricas diretas e indiretas (por 100 mil nascidos vivos), ocorridos na regiáo metropolitana I do estado do Pará, no período de janeiro de 2013 a dezembro de 2015.

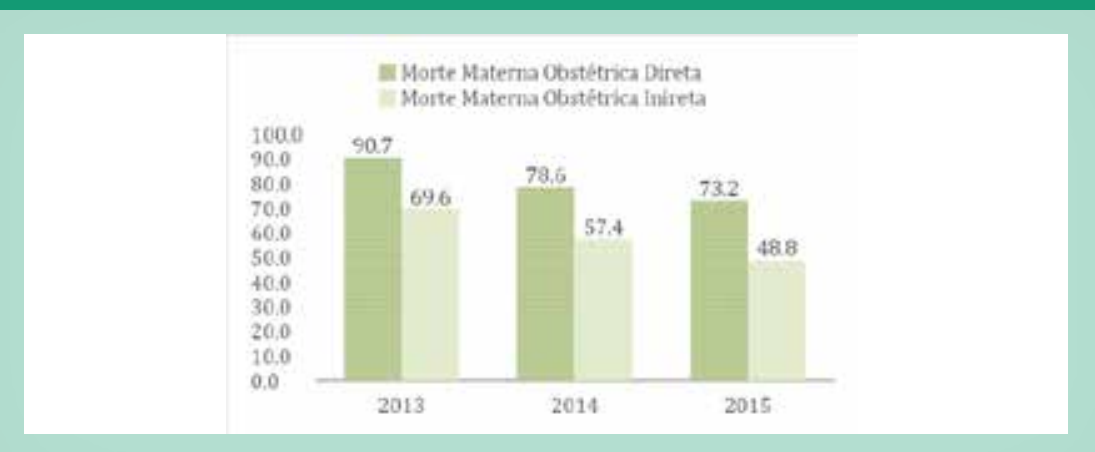

\section{RESULTADOS}

Após a coleta no SIM foi possível identificar que ocorreram 53 óbitos no ano de 2013, 45 óbitos no ano de 2014 e 40 óbitos no ano de 2015, e a partir do número de nascidos vivos nos respectivos anos foi possível determinar a Razão de Mortalidade Materna (RMM) que segue descritos na tabela 1:0

Observa-se um decréscimo anual da RMM no período de estudo. Considerando o triênio a RMM foi de 139,4 óbitos a cada 100 mil nascidos vivos. A figura 01 mostra a discreta redução da RMM.

Considerando a RMM por causas obstétricas, foi possível identificar que predominaram as causas obstétricas diretas, com RMM de 90,7 em 2013, de78,6 em 2014 e de73,2 em 2015 um pouco a mais do que a RMM das causas obstétricas indiretas $(69,6 ; 57,4$ e 48,8/100.000nv em cada período), conforme é mostrado na tabela 2, apresentando ainda uma variação 15,19\% (2014-2013); de 10,32\% (2015-2014) e uma variação significativa de 23,94\% quando comparado de 2015-2013.

Na figura 02, observa-se um declínio da RMM tanto por causas diretas como por causas indiretas. Já na relação entre as causas diretas sobre as indiretas se observa uma ascensão dos casos de 0,9 em 2013, elevou para 1,4 em 2014 e 1,5 em 2015.

\section{DISCUSSÃO}

A mortalidade materna pode ser estratificada em baixa, média, alta e muito alta, segundo suas razões (menores que 20, entre 21 e 49, entre 50 e 149 e maior que 150 , respectivamente) $)^{3}$.

A RMM observada no presente estudo foi muito alta no ano de 2013 e alta nos anos de 2014 e 2015, observando-se uma nítida redução nos dois últimos anos (Figura 01), ou seja, níveis acima de 100 óbitos a cada 100.000 nv, em ambos períodos estudados, o equiva- 
lente a cinco vezes acima do que seria recomendado.

Botelho $^{6}$, em estudo realizado no estado do Pará, tendo como base os registros do SIM e do SISNAC, encontrou uma RMM de 50,4 em 2010, de 50,1 em 2009 e de 52 em 2008; dados esses inferiores aos observados no presente estudo, mostrando uma preocupante ascendência da mortalidade materna em nosso meio.

Em outro estudo similar a este, realizado no Maranhão, no período de 2006 a 2010 encontrou-se uma RMM de 94,4 óbitos para cada 100.000 nascidos vivos ${ }^{7}$. Dados alarmantes, mais abaixo do que foi observado no presente estudo.

Ferraz e Bordignon ${ }^{8}$ destaca que o Brasil apresentou um aumento 11,92\% na RMM entre os anos de 2000 a 2009. Um aumento que foi diferente para cada região tendo o Norte apresentado um crescimento de 15,46\%; o Nordeste, $18,53 \%$; o Sudeste, $10,31 \%$; e o Centro-Oeste, 50,54\% no número absoluto de mortes maternas. Em contrapartida, o Sul apresentou uma redução, em números absolutos, correspondente a $15,76 \%{ }^{8}$.

Ainda nesse mesmo estudo foi possível constatar que no intervalo de 10 anos (2000- 2009), ocorreram 16.520 óbitos maternos no Brasil, com uma razão de mortalidade materna de 54,83 óbitos a cada 100 mil nascidos vivos nesse período. Esse índice de mortalidade materna brasileira também evidencia os números elevados que deveriam estar diminuindo ${ }^{8}$.

De acordo com o estudo realizado pela World Health Organization (WHO), em conjunto com o United Nations International Children's EmergencyFund (UNICEF), em 1990, estimou-se que aproximadamente 585.000 mulheres, em todo o mundo, morreriam vítimas de complicações ligadas ao ciclo gravídico-puerperal, sendo 99\% dessas em países do chamado "Terceiro Mundo", o que sugere a morte ma-

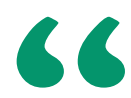

Ainda nesse mesmo estudo foi possível constatar que no intervalo de 10 anos (20002009), ocorreram 16.520 óbitos maternos no Brasil, com uma razão de mortalidade materna de 54,83 óbitos a cada 100 mil nascidos vivos nesse período. Esse índice de mortalidade materna brasileira também evidencia os números elevados que deveriam estar diminuindo ${ }^{8}$. terna como um indicador sensível das desigualdades sociais mundialmente existentes. Destaca-se que não há outro indicador de saúde pública que mostre maior disparidade entre os países, em função de sua riqueza e desenvolvimento, que a mortalidade materna ${ }^{9}$. Ou seja, a RMM brasileira ainda está muito distante do que o recomendado para os anos de 2015 conforme era o objetivo do Pacto do Milênio.

Considerando o comportamento da mortalidade materna no Brasil, a distribuição da RMM é bastante heterogênea segundo as distintas regiões da Federação. Estudos apontam que as maiores razões são encontradas para as regiões Norte, Nordeste e Centro-Oeste, as mais pobres do país. E as menores para as regiões Sul e Sudeste, descritas como detentoras de melhores condições de desenvolvimento socioeconômico ${ }^{10}$, corroborando com o que foi encontrado no presente estudo.

Estudo realizado pela OMS, UNICEF, UNPFA e Banco Mundial estimou que em 2015 aproximadamente 303 mil mulheres em todo o mundo morreriam vítimas de complicações ligadas ao ciclo gravídico-puerperal. A RRM nos países em desenvolvimento em 2015 foi de 239 por 100 mil nascidos vivos contra 12 por 100 mil nascidos vivos em países desenvolvidos ${ }^{11}$.

Nas Américas, existe contraste expressivo entre os países desenvolvidos e em desenvolvimento no risco de morte materna. A RMM nos Estados Unidos foi de 12 e 14 óbitos maternos por 100 mil NV, nos anos 1990 e 2015, respectivamente; no Canadá foi sete e sete nos mesmos anos. Embora observado aumento da RMM nos USA, o risco permanece baixo. Em países com dificuldades de desenvolvimento econômico, a RMM diminuiu nas últimas décadas, porém se mantendo em valores elevados, como a Bolívia que caiu de 425 óbitos maternos por 100 mil NV, em 1990, para 206, em 2015; o Haiti passou de 625 para 359, nos mesmos anos ${ }^{11}$. 
Ainda relacionado a RMM, o presente estudo apresenta níveis elevados para os óbitos por causas obstétricas diretas, óbitos esses que em sua grande maioria poderiam ter sido contornados ainda no pré-natal (figura 02), contudo, ambas as causas (direta e indireta) ainda perdura acima do que é recomendado pela OMS

Resultado esse que se assemelhou em um estudo realizado no Paraná no qual também predominaram as causas obstétricas diretas, apresentando uma RMM de 42/100.000 nv, duas vezes superior à RMM das causas obstétricas indiretas $(16 / 100.000 \mathrm{nv})^{12}$, coincidindo com o que foi encontrado no presente estudo, no qual apresenta números elevados de causas obstétricas diretas, contudo vem ocorrendo uma discreta redução nos últimos anos.

Ao realizar a relação entre as causas diretas sobre as indiretas encontradas nesta pesquisa, se observa uma ascendência de 0,9 em 2013, elevou para 1,4 em 2014 e 1,5 em 2015. Resultado este que foi o oposto ao encontrado na pesquisa realizada por Mendes (2013) em São Paulo, onde foi observado que a relação entre as causas diretas sobre as indiretas caiu de 2,8 em 2000 para 1,7 em 2011, valores superiores se comparado a este estudo, e inferior ao nacional. No Brasil, a relação entre as causas era de 3,5 vezes em 2000, e reduziu-se para 2,4 vezes, em 2010. O aumento de proporção de causas indiretas indica uma provável melhoria nas investigações dos óbitos suspeitos ${ }^{13,14}$.

\section{CONCLUSÃO}

A Razão de Mortalidade Materna aqui encontrada, ainda se encontra muito aquém do desejável, onde representa 160,3 óbitos a cada 100 mil nascidos vivos no ano de 2013,136,0 em 2014 e 121,9 em 2015 e ao relacionar com o esperado pela OMS que seria de 10 a 20 óbitos a cada 100 mil nascido vivo, é possível ver a discrepância e a necessidade de que ainda se tem muito por fazer em prol da saúde da mulher.

Esses resultados confirmam que a RMM também é considerada um importante indicador da realidade social. Regiões mais desenvolvidas, com assistência à saúde de qualidade e políticas públicas eficazes se destacam com as menores razões. Dessa forma, pode se supor que as condições pelas quais as mulheres morrem espelham o nível do desenvolvimento humano da população, assim um alto grau de mortalidade materna, como foi identificado no Pará, é indicativo de precárias condições socioeconômicas, que culminam com dificuldades de acesso aos serviços de saúde.

Além disso, é necessário enfatizar que não foi observada redução estatisticamente significativa da RMM no Estado do Pará nos últimos anos. O que implica no questionamento sobre a eficácia das medidas que estão sendo realizadas para diminuir a mortalidade materna. Esse resultado vem ainda confirmar a permanência de importantes entraves que impossibilitam a redução da RMM no estado; entre os quais estão a carência do planejamento familiar, a assistência pré-natal inadequada, a falta de vaga no serviço hospitalar e a deficiência de estrutura dos hospitais, que acarretam precariedade no atendimento às gestantes, sendo assim, segue o enorme desafio em se alcançar os objetivos do milênio em reduzir a ocorrência de óbitos materno

\section{Referências}

1. Matos, J.C. Mortalidade por aborto no Estado do Paraná: 1998 a 2004. Revista Eletronica de Enfermagem; 2007.v.9, n.3, p.80614 , set.- dez.

2. Laurenti, R. A mortalidade materna nas capitais brasileiras: algumas características e estimativa de um fator de ajuste. Revista Brasileira de Epidemiologia; 2004.v.7, n.4, p.449-60.

3. OMS - World Health Organization. The Millennium Development Goals Report 2014. Disponivelem: <http://www.un.org/ millenniumgoals $>$ (Acessado em SET/2014).

4. Brasil, Ministério da Saúde. Manual dos Comitês de Mortalidade Materna. 3a ed. Brasília: MS; 2007.

5. Mota, S.M.M; Gama, S.G.N; Theme Filha, M.M. A investigação do óbito de mulher em idade fértil para estimar a mortalidade materna no Município de Belém, Estado do Pará, Brasil. Epidemiologia ServiçoSaúde.Brasília, 18(1):55-64, jan-mar, 2009.

6. Botelho, N.M; Silva, I.F,M,M; Tavares, J.R; Lima, L.O. Causas de mortes maternas no estado do Pará, Brasil. Revista Brasileira de Ginecologia e Obstetrícia; 2014.

7. Portela, N. L. C.; Araújo, N. J.; Monte, L. R. S. Mortalidade materna no estado do Maranhão no período de 2006 a 2010. R. Interd. v. 8, n. 3, p. 75-82, jul. ago. set. 2015

8. Ferraz, L; Bordignon, M. Mortalidade Materna no Brasil: Uma realidade que precisa melhorar. Revista Baiana de Saúde Pública. 2012; v.36, n.2, p.527-538, abr/jun,

9. Sousa, M. H. Sistemas de informação em saúde e monitoramento de morbidade materna grave e mortalidade materna. Rev. Bras. Saude Mater. Infant, Recife, v. 6, n. 2, abr./jun. 2006. 10. Brasil. Ministério da Saúde. Manual dos comitês de mortalidade materna. Brasília: Ministério da Saúde; $1994.119 \mathrm{p}$

11. WHO - WORLD HEALTH ORGANIZATION. Strategiestowardendingpreventable maternal mortality (EPMM) [Internet]. 2015 [acessado $15 \mathrm{fev}$ 2016]. Disponível em: <who.int/reproductivehealth/topics/maternal_perinatal/epmm/en/>

12. Soares, Vânia Muniz Néquer. Causas de mortalidade materna segundo níveis de complexidade hospitalar. Rev. Bras. Ginecol. Obstet., Rio de Janeiro, v. 34, n. 12, p. 536-543, Dec. 2012. 13. Mendes, José Dínio Vaz; Osiano, Vera Lucia Rodrigues Lopes. A Mortalidade Materna no Estado de São Paulo, Brasil. BEPA, Bol. epidemiol. paulo (Online), São Paulo, v. 10, n. 114, jun. 2013

14. Brasil. Ministério da Saúde. Óbitos de mulheres em idade fértil e óbitos maternos - Brasil. Brasília, DF: DATASUS, 2016. Disponível em: < http://tabnet.datasus.gov.br/cgi/tabcgi.exe?sim/cnv/mat10uf.def>. Acesso em: 14 dez. 2016. 